\title{
Cellular Plant Responses: Plant-Microbe Interactions
}

\author{
Bhandari P.A. ${ }^{1}$, Patil A.G. ${ }^{2}$, Ojha M.D. ${ }^{2}$, Pawar S.S. ${ }^{3}$, Adhikari P.R ${ }^{3}$. \\ ${ }^{1}$ P.T.V.A's Sathaye College, Vile Parle (E), Mumbai-400 057, India \\ ${ }^{2}$ V.E.S., College of Arts, Science and Commerce, Chembur, Mumbai-400 071, India \\ ${ }^{3}$ Padmashree Dr. D.Y. Patil University, Navi Mumbai, 400614, India
}

\begin{abstract}
Soil-borne microorganisms can induce an enhanced defensive capacity in above-ground plant parts, which provides protection against a broad spectrum of microbial pathogens; even insect herbivores and emerged as important regulators of this induced systemic resistance.
\end{abstract}

Keywords- Plant Responses, Plant-Microbe Interactions, Functional analysis

\begin{abstract}
Introduction
Encounters between plant cells and both 'friendly' and 'hostile' microbes (such as those in symbiotic and pathogenic interactions, respectively) trigger a range of highly dynamic plant cellular responses. Densely colonized soil contains beneficial mycorrhizal fungi and rhizobia, which associate with roots and provide plants with mineral nutrients and fixed nitrogen, respectively, in exchange for carbon. By contrast, plants are constantly exposed to a range of fungal, bacterial and viral pathogens, and have evolved unique defense mechanisms to fight these infections. These include reorganization of the cytoskeleton, organelle translocation, vesicle trafficking, and alterations in subcellular protein localization. Recent progress in this border-land that bridges the fields of plant-microbe interactions and cell biology heralds the transition from descriptive phenomenology to the identification and characterization of key molecules that are involved in these processes. Intriguingly, molecular events that occur in plant cells in response to microbes also take place upon abiotic wounding and during fundamental plant developmental processes, such as the tip growth of pollen, root hairs and trichomes. Thus, elementary 'activity modules' that are required for the generation of cell polarity in plant morphogenesis appear to be re-used in both abiotic and biotic stress response pathways.
\end{abstract}

\section{Dynamic Subcellular Responses In Plants A. Background}

Application of latest tools to investigate cell responses in vivo [1] includes use of fluorescent protein tags- green fluorescent protein (GFP) and its derivatives as live cell markers in model plants [2]. They highlight the pivotal role played by epidermal cells, as the first site at which direct cell-to-cell contact takes place.

\section{B. Survey of early plant-cell responses} Cellular responses that take place between the perception of a microorganism (either via its secreted elicitors or upon direct contact) and determination of fate of the interaction is summarized below [3].

\section{i. Online booking: host cell pre-alert}

Chemistry is the language of cells, and plantinteracting microbes are no exception. Any plantmicrobe interaction with chemical signaling, on investigation revealed the existence of a precise molecular dialogue prior to and during direct cellto-cell contact $[4,5,6]$. Pathogenic fungal effector molecules are reported to trigger signaling cascades [7], gene activation [8], the release of defense-related molecules, and systemic reactions [9]. In symbioses, root nodulation is the most-studied system from this point of view [10]. Isolated chemical signals released by rhizobia, the so-called Nod factors [11], induce a number of rapid molecular or cellular responses in root hairs of compatible plant species, including the activation of specific intracellular signaling pathways leading to gene activation [12, 13]. Additional later responses to Nod factors include the initiation of nodule organogenesis in the inner cortex [14]. In the early 1990's, Heidstra and coworkers (1994) described the swelling of roothair tips exposed to a Nod factor solution, later correlated with a transient fragmentation and reorganization of the apical cytoskeleton $[15,16]$. Recently, it has been shown that exogenous application of Nod factors to root hairs results in the rapid fragmentation of actin bundles and is accompanied by increased apical influxes and intracellular levels of calcium [17, 20, 21]. Chemical signals released from AM fungi: activation of early nodulin gene expression [18] and signal transduction-related gene expression [19] has been reported in the presence of permeable membranes that block direct fungal contact with the root. Olah and associates (2005) described an increase in the frequency of root branching in response to diffusible AM fungal factors. Together, such observations support the idea that diffusible microbe signals induce a first level of basic plant responses, ranging from a general pre-alert to more obvious morphological responses at a molecular, cellular, and organ level.

\section{ii. Boarding gate: direct cell contact}

Plant non-host resistance strategies represent the first line of defense against invading microbes [22]. Surveillance system that allows plants to 
recognize and effectively respond to most 'wanna-be' pathogens, a phenomenon frequently termed 'non-host resistance'. Despite the fundamentally different final outcome of microbial contacts, plant cells exhibit a surprisingly similar set of highly dynamic cellular responses during contacts. These activities ultimately lead to extensive polarization towards the microbe at the single-cell level [23]. Among subcellular responses, the 1st visible reaction is rapid translocation of the cytosol and subcellular elements to the contact site. This is called cytoplasmic aggregation (CA) [25]. CA results from the establishment of a striking asymmetry within the cell [23] through rapid site-directed cytoplasmic streaming [25]. CA depends primarily on the action of the actin cytoskeleton [26, 27]. Recent cytochemical and GFP-labeling of actin microfilaments have revealed their radial organization around the penetration site in many plant-pathogen interactions [24]; while microtubules show more heterogeneous patterns in barley and flax [27], in Soybean [29], in Arabidopsis [30]. Most organelles assemble at the potential infection site, where they contribute to an intense secretory activity [31], reinforcing the wall with compounds that improve its resistance to the chemical and physical attack directed by the pathogen [32]. Cell-wall appositions, or papillae, develop around and in front of fungal appressoria, intercellular hyphae, and penetration pegs [32]. Secretion of antifungal compounds has been proposed by Stein and colleagues (2006). When cell-wall appositions are not sufficient to arrest noncompatible pathogens or their effectors from reaching the cytoplasm [33], a second line of defense can be initiated. Specific receptors trigger signaling cascades that program cell death [34].

\section{Comparative analysis of host cell responses}

i. Host vesicle transport and secretion

Members of the superfamily of soluble Nethylmaleimide- sensitive factor adaptor protein receptor (SNARE) polypeptides contribute to limiting host cell entry by powdery mildew fungi both in monocot and dicot plant species. Mutations in the gene encoding plasma membrane (PM)-resident Arabidopsis AtPEN1 target membrane SNARE (t-SNARE, also referred to as syntaxin) allow enhanced cell invasion by the grass powdery mildew fungus Blumeria graminis f. sp. hordei (Bgh) [36]; suggesting that t-SNARE function is essential to arrest fungal ingress at the cell periphery [36, 37, 38]. Further SNARE protein, SNAP-25, is known to form binary complexes with syntaxins to promote membrane-fusion events in animal and yeast cells [39]. Indirect evidence for a role of exocytosis in non-host resistance - Secretion of a cocktail of metabolites that have antimicrobial activity confers tissue-specific resistance to a range of bacterial microbes in the roots of Arabidopsis. Activation of the secretory pathway represents systemic acquired resistance (SAR), a plant-wide immune response that is triggered upon a local stimulation of the plant's pathogen surveillance system.

\section{ii. Reorganization of the plant cytoskeleton and organelle positioning}

Numerous pharmacological inhibitor studies have demonstrated the importance of cytoskeletal rearrangements for the execution of pathogen entry control at the cell periphery [23]. Actin microfilaments play a pivotal role in timely and spatial recruitment of the plant's defensive forces at infection sites [30]. Dynamics of cytoskeleton and endomembrane reorganization during pathogen attack have been explored in living cells through studies of GFP-tagged cell components. E.g., extensive changes in the distribution and morphology of the ER followed during a 15-minute period from the onset of reorganization beneath a Phytophthora sojae hypha growing across the outer epidermal cell wall [40]. Although experiments corroborate the importance of adaptive cytoskeleton remodelling in potential pathogens, molecules that signal and carry out dramatic reorganizations are still elusive. Possible role for RAC/ROP family $G$ proteins: potent regulators of pathogen-induced plant microfilament reassembly that might be targeted by fungal pathogens to establish compatibility. Another plant susceptibility factor, barley MLO protein, also affects actin-dependent control of pathogen entry at the cell periphery. Symbiotic relationships with mycorrhizal fungi and rhizobium is accompanied by dramatic cytological modifications - cytoskeleton reorganization, modifications in the root hair and cortex cells of the host plant for root hair curling, infection thread growth and root nodule development [24]. In summary, host cytoskeleton plays a key regulatory role in biotic interactions with both pathogenic and beneficial microorganisms. As a consequence, diverse strategies to interfere with the plant's cytoskeleton by delivery of specific proteinaceous or metabolic effector molecules appear to have evolved independently in the different microbial taxa. Nuclear movement in response to a threat is seen, however it moves along with all surrounding organelles [25, 40]. Concentrating them all at one site possibly shortens response time to stimulus, in a cell in which communication and transport must circumvent the huge central vacuole. Thanks to local repositioning, signal transduction pathways may activate gene expression more rapidly and transcripts can be concentrated at the site where they are translated into key response proteins [42]. Lastly, from an evolutionary point of view, cell mechanisms set in 
motion by these plant-microbe interactions are probably related to a more archaic cellular process. Focused secretion, cytoskeleton mobilization, nuclear movement, cytoplasmic strands crossing the vacuole-all of these responses are directly involved in cell division, thus supporting the hypothesis that basic cell processes such as cytokinesis may have been recruited and modulated as the need arose to reinforce the cell wall or accommodate a biotroph within the cell lumen [75].

\section{iii. Dynamic changes in sub-cellular protein localization}

Identification of individual proteins that exhibit an altered subcellular localization upon a biotic stimulus is a fresh finding. Application of a peptide elicitor, Pep13, derived from the oomycete pathogen Phythophtora triggers an innate immune response in cultured parsley cells that involves the activation of three mitogen activated protein kinases (MAPKs); immunolabelling, all 3 MAPKs exhibits an increase in nuclear localization upon activation [42].

\section{iv. Polarizing the host cell}

Hypotheses postulated concerning the way a cell perceives contact with a potential guest. Hypothetical mechanisms proposed that involve organization of specialized membrane domains [43], which label the contact site, and focal accumulation of membrane-bound proteins has been demonstrated by yellow fluorescent protein tagging [44].

\section{Cytoskeleton and Cellular Response Targets I. Cytoskeleton functions in plant-microbe interactions}

Plant cytoskeleton contributes to establishment of cell polarity during plant development and morphogenesis. Rapid changes in regular cytoskeleton architecture occur upon contact of individual plant cells with both pathogenic and symbiotic microbes. In case of pathogens, polarized cytoskeletal rearrangements are thought to allow localized delivery of cargo for defense execution, while in symbiotic interactions the reorganization may advance establishment of the symbiotic relationship. Microbial metabolites and effector proteins are released into plant cells for manipulation of the host cytoskeleton, while some secreted plant defensive polypeptides may target the microbial cytoskeleton. The cytoskeleton thus emerges as a potential mutual target in plant-pathogen combats that appears to be under attack by effector molecules from both sides [73].

\section{Regulator and target of biotic interactions in plants}

Plant cytoskeleton composed of microtubules and actin microfilaments, plays role in plant cell growth and development and intracellular organization and motility [24, 46, 47].

\section{a. Response to pathogenic fungi and oomycetes}

Cytoplasmic aggregation is an example of site directed cytoplasmic streaming and is dependent upon the action of the actin component of the cytoskeleton, shown using inhibitors of actin polymerization [48, 49]. Assessment of the contribution made by cytoplasmic aggregation to plant resistance may be complicated by an influence of the pathogen on host cell structure and metabolism. As well as redirection by the plant for defense purposes, redeployment of plant materials via cytoskeletal rearrangements could also be orchestrated by an invading pathogen as part of its strategy to obtain nutrients from the plant.

\section{b. Plant cytoskeletal response to mycorrhizal fungi}

Two main categories of mycorrhizal symbiosis: ectomycorrhizal and endomycorrhizal associations. Ectomycorrhizal fungi do not penetrate host cell wall but form a dense layer, called the mantle, on surface of the root and a network of intercellular hyphae within the root tissues. In endomycorrhizae, fungal cells penetrate the plant cell wall and elaborate specialized infection structures within the plant cell; although, they remain surrounded and separated from the host cytoplasm by an intact plant plasma membrane. Increases in expression of tubulin genes and concentrations of plant a-, b, and g-tubulins and actin have been observed in a variety of ecto- and endomycorrhizas [48-52]. Studies of cytoskeletal behavior in ectomycorrhizal associations are limited. In both cases, after fungal invasion, plant microtubules and actin microfilaments disappear almost entirely from the plant cell cortex and assemble close to the surface of the trunk hyphae, arbuscules [53-57].

\section{c. Plant cytoskeleton targeted by pathogenic bacteria}

Rhizobia and many plant and animal bacterial pathogens possess the type-III secretion system that injects bacterial proteins (effectors) into the host cytoplasm [58, 59]. In animals, the host cytoskeleton, in particular actin microfilaments, is a major target of type-III effectors for pathogen virulence [58-61]. Various type-III effectors of plant pathogenic bacteria are virulence factors that suppress plant defense responses such as hypersensitive cell death and expression of defense genes [62-64]. Till date, no reports of 
type III effectors targeting plant cytoskeletal elements exist.

\section{d. Concluding remarks}

In most plant-microbe interactions, the development of structured cytoskeletal arrays at the interaction site is associated with outcomes that are beneficial to the plant. Examples include the radial arrays of actin microfilaments and microtubules that accompany cytoplasmic aggregation during defense responses against invading pathogens, the dense network of actin microfilaments and microtubules that forms around arbuscules of mycorrhizal fungi, and the lattice of actin microfilaments or radial array of microtubules found among symbiosomes in root nodules. On the other hand, degradation of the plant cytoskeleton is often associated with outcomes of plant interactions with other organisms that are detrimental to the plant. These observations underscore the important role played by the plant cytoskeleton in mediating the plant cell's response to biotic factors, e.g., in forming an apoplastic barrier to arrest pathogen ingress. Changes in cytoskeletal organization may also facilitate signaling of the presence of symbionts or pathogens on the plant surface. There is growing evidence that actin and microtubule arrays in plant cells participate in signaling cascades initiated at the plasma membrane, enabling adaption to environmental factors.

\section{Root hair response \\ Symbiotic relationship with rhizobia}

Changes in cytoskeletal arrays in root hairs and cortical cells occur during the establishment of a symbiotic relationship with certain gram-negative bacteria collectively called rhizobia, and it is clear that microtubules and actin microfilaments play active and necessary roles in root hair curling, growth of the infection thread and root nodule development [24].

\section{Root hairs following inoculation}

Rhizobial attachment, or application of hostspecific nodulation (Nod) factors [65, 66] causes a localized influx of calcium, depolarization of the plant plasma membrane, alkalinization of the cytoplasm, and curling (or deformation) of the hair [65, 67-68]. Chalcone synthase (CHS) of Vigna unguiculata is encoded by a gene family that is abundantly transcribed in leaves and nodules. Inoculation with Rhizobium sp. NGR234, which nodulates $V$. unguiculata, or with NGRDnodABC, a mutant deficient in Nod factor production, induced rapid accumulation of $\mathrm{CHS}$ mRNAs in roots and root hairs. As both Nod+ and Nod- bacteria provoke responses, induction of CHS gene expression may involve symbiotic or defense responses. Four days after inoculation with the wild-type Rhizobium sp., the transcript levels increased in roots but decreased in root hairs. Use of a region unique to the $5 c$ end of a specific CHS gene (VuCHS1) showed that increases of transcript levels in root hairs $24 \mathrm{~h}$ after inoculation with both rhizobia were specific to this gene. Transcripts of this gene in roots were only detectable 4 days after treatment with NGR234. It is possible therefore that accumulation of VuCHS1 follows the infection pathway of rhizobia entering legume roots. Purified Nod factors induced accumulation of transcripts, showing that they might be part of the signal transduction pathway leading to $\mathrm{CHS}$ expression [69]. Response of the actin cytoskeleton in vetch root hairs after application of host-specific Nod factor is studied. Within 3 to $15 \mathrm{~min}$, the number of sub-apical fine bundles of actin filaments (FB-actin) increased in all developmental stages. Tip growth resumed only in hairs in which the FB-actin density and the length of the region with FB-actin exceeded a minimal value. If the fine bundles of actin microfilaments in the tip of the hair are depolymerized by treatment with cytochalasin D, deformation but not new outgrowth occurs. These results are consistent with a role of the subapical actin microfilaments in targeting vesicle exocytosis to the growing tip of the hair [71].

\footnotetext{
Functional analysis of sensing receptor and domains I. Intracellular dynamics

Detection of potentially infectious microorganisms is essential for plant immunity. Microbial communities growing on plant surfaces are constantly monitored according to their conserved microbe-associated molecular patterns (MAMPs). In recent years, several pattern-recognition receptors, including receptorlike kinases and receptor-like proteins and their contribution to disease resistance have been described [74]. Plants are able to specifically recognize microbial effector molecules via nucleotide-binding site leucine rich repeat receptors (NB-LRR). A number of recent studies show that NB-LRR translocate to the nucleus in order to exert their activity. Current knowledge regarding the recognition of MAMPs by surface receptors, receptor activation, signaling, and subcellular redistribution are discussed. MAMPs are recognized by cognate pattern recognition receptors (PRR) that trigger immediate defense responses leading to basal or nonhost resistance, or MAMP- or PAMP-triggered immunity [75]. To date, all known PRR in plants are plasma membrane-resident proteins, allowing the perception of MAMPs to occur at the cell surface. In contrast to PRR, receptor proteins recognizing effectors are found predominantly in the cytoplasm, although a few are surface receptors.
} 


\section{Microbial patterns}

Typically, MAMPs represent structures that are essential for microbial life. The elicitor active epitope within flagellin flg22 was identified from the conserved $\mathrm{N}$-terminus. Moreover, two proteins normally located in the bacterial cytoplasm, namely cold shock protein (CSP) and elongation factor Tu (EF-Tu), trigger immune responses in members of Solanaceae and Brassicaceae.

\section{Receptors of microbial patterns}

Receptors detecting microbial patterns can be divided into surface and intracellular receptors. The latter are the nucleotide- binding site (NB) leucine-rich repeat (LRR) class of receptors, later referred to as NB-LRR. Surface receptors are known to detect both MAMPs and effectors and include receptor-like kinases (RLK), receptor-like proteins (RLP) and extracellular binding proteins. RLK reside in plasma membranes and are composed of a putative extracellular ligandbinding domain, a single transmembrane domain, and an intracellular serine/ threonine kinase domain. Thus, RLK are proteins with a "receptor" and a "signaling" domain in one molecule, and appear to be structurally related to animal receptor-tyrosine kinases (RTKs. Similarly, RLP consist of an extracellular domain and a membrane-spanning domain. However, they lack an intracellular activation domain and, consequently, require interaction with adaptor molecules for signal transduction. Many Arabidopsis genes encoding RLK and RLP were found to be induced upon flg22 or EF-Tu treatment, suggesting that they may function as immune receptors.

\section{Microbial and endogenous patterns by RLK}

Pattern-recognition of symbiotic bacteria was shown to be mediated by two RLK identified from Lotus japonicus, NFR1 and NFR5, that contain two and three LysM motifs, respectively.

\section{RLP and extracellular binding proteins}

Recently, a high-affinity binding protein for fungal-derived chitin-fragments, CEBiP, has been isolated from rice. It is predicted to have two LysM domains in its extracellular part and a single transmembrane domain. Furthermore, it appears to be highly glycosylated. CEBiP was shown to mediate binding to chitin octamers and to be responsible for chitin- triggered immune responses. Thus, CEBiP is a prime candidate for being a chitin receptor. In Arabidopsis, one of the LysM-RLK that is trancriptionally induced by flg22 or elf18 might perform this function. The ability to monitor microbial growth at the cell surface is pivotal for plant fitness. Devoid of an antigenpresenting adaptive immune system, plants have evolved a large repertoire of potential immune receptors that recognize MAMPs or mediate effector perception. The few MAMP receptors identified in plants are all surface receptors that physically interact with their cognate ligands. However, the MAMP binding domains of plant PRR remain to be characterized. Moreover, the crystal structures of RLK and RLP are required for a detailed understanding of molecular changes during receptor activation.

\section{Molecular Characterization and Functional Analysis}

Analysis of the fully sequenced genome of the wheat leaf-specific fungal pathogen Mycosphaerella graminicola identified only a single gene encoding a member of the necrosisand ethylene-inducing peptide 1 (Nep1)-like protein family (NLP). NLP proteins have frequently been shown to trigger cell death and activation of defense signaling reactions in dicotyledonous plants. However, complete lossof-function reverse genetics analyses for their importance in the virulence of eukaryotic plant pathogens are generally lacking. Real-time quantitative polymerase chain reaction on MgNLP demonstrated the gene to be specifically expressed in planta. Peak expression was observed during the immediate presymptomatic phase of colonization of a susceptible host genotype. This was followed by a dramatic decrease during disease lesion formation which, in this system, exhibits characteristics of host programmed cell death (PCD). No comparable peak in transcript levels was seen during an incompatible interaction with a host genotype exhibiting gene-for-gene-based disease resistance. Heterologously expressed MgNLP protein induced necrotic cell death and the activation of defense-related genes when infiltrated into Arabidopsis leaves but not in leaves of a susceptible wheat genotype. MgNLP infiltration also failed to stimulate wheat mitogenactivated protein kinase activities. Finally, targeted deletion of $M$. graminicola MgNLP caused no detectable reduction in plant pathogenicity or virulence, suggesting that this protein is not a major virulence determinant during fungal infection of its host plant. This represents the first complete loss-of-function analysis of NLP in a eukaryotic plant pathogen and findings in the context of possible functions for NLP in pathogens which only infect monocotyledonous plants [76].

\section{References}

[1] Dhanoa P. K., Sinclair A. M., Mullen R. T. and Mathur J. (2006) Can. J. Bot., 84:515-522.

[2] Brandizzi F., Fricker M. and Hawes C. (2002) Nat. Rev., 3:520-530.

[3] Genre A. and Bonfante P. (2007) MPMI., 20(9):1023-1030. 
[4] Glazebrook J. (2005) Annu Rev Phytopathol., 43:205-27.

[5] Geurts R., Fedorova E. and Bisseling T. (2005) Curr. Opin. Plant Biol., 8:346352.

[6] Paszkowsky U. (2006) New Phytol., 172 :35-46.

[7] Zhang S. and Klessig D. F. (2001) Trends Plant Sci., 6:520-527.

[8] Schenk P. M., Kazan K., Wilson I., Anderson J. P., Richmond T., Somerville S. T. and Manners J. M. (2000) Proc. Natl.Acad. Sci. U.S.A., 97:11655-11660.

[9] Grant M. and Lamb C. (2006) Curr Opin Plant Biol., 9:414-420.

[10] Gage D. J. (2004) Microbio. Mol Biol Rev., 68:280-300

[11] Truchet G., Roche P., Lerouge P., Vasse J., Camut S., Debilly F., Prome J. C. and Denarie J. (1991) Nature 351:670-673.

[12] Lohar D. P., Sharopova N., Endre G., Peñuela S., Samac D., Town C., Silverstein K. A. T. and VandenBosch K. A. (2006) Plant Physiol., 140:221-234.

[13] Oldroyd G. E. D. and Downie J. A. (2004) Nat. Rev., 5:566-576.

[14] Timmers A. C., Auriac M. C. and Truchet G. (1999) Development, 126:3617-3628.

[15] Cárdenas L., Vidali L., Dominguez J., Perez H., Sanchez F., Hepler P. K. and Quinto C. (1998) Plant Physiol., 116:871-877.

[16] De Ruijter N. C. A., Bisseling T. and Emons A. M. C. (1999) Mol. Plant-Microbe Interact., 12:829-832.

[17] Vassileva V. N., Kouchi H. and Ridgea R. W. (2005) Plant Cell, 17:1777-1787.

[18] Esseling J. J., Lhuissier F. G. and Emons A. M. (2003) Plant Physiol., 132:19821988.

[19] Kosuta S., Chabaud M., Lougnon G., Gough C., Dénarie J., Barker D. G. and Bécard G. (2003) Plant Physiol., 131:111.

[20] Weidmann S., Sanchez L., Descombin J., Chatagnier O., Gianinazzi S. and Gianinazzi-Pearson V. (2004) Mol PlantMicrobe Interact., 17:1385-1393.

[21] Sanders D., Pelloux J., Brownlee, C. and Harper J. F. (2002) Plant Cell Suppl., 2002:S401-S417.

[22] Miwa H., Sun J., Oldroyd G. E. D. and Downie A. J. (2006) Plant J., 48:883894.

[23] Schmelzer E. (2002) Trends Plant Sci., 7:411-415.

[24] Takemoto D. and Hardham A. R. (2004) Plant Physiol., 136:3864-3876.

[25] Heath M. C., Nimchuk Z. L. and Xu H. (1997) New Phytol., 135:689-700.

[26] Hazen B. E. and Bushnell W. R. (1983) Physiol Plant. Pathol., 23:421-438.
[27] Tomiyama K., Sato K. and Doke N. (1982) Ann Phytopathol. Soc. Jpn., 48:228230.

[28] Kobayashi I., Kobayashi Y. and Hardham A. R. (1994) Planta, 195:237-247.

[29] Cahill D., Rookes J., Michalczyk A., McDonald K. and Drake A. (2002) Plant Pathol., 51:629- 640.

[30] Takemoto D., Jones D. A. and Hardham A. R. (2003) Plant J., 33:775-792.

[31] Thordal-Christensen H. (2003) Curr Opin Plant Biol., 6:351-357.

[32] Zeyen R. J., Carver T. L. W. and Lyngkjaer M. F. (2002) Epidermal cellpapillae, 107-125.

[33] Aist J. R. (1976) Annu Rev Phytopathol., 14:145-163.

[34] O'Connell R. J. and Panstruga R. (2006) New Phytol., 171:699-718.

[35] Dangl J. L. and Jones J. D. G. (2001) Nature 411:826-833.

[36] Assaad F. F., Qiu J. L., Youngs H., Ehrhardt D., Zimmerli L., Kalde M., Wanner G., Peck S. C., Edwards H. and Ramonell K. (2004) Mol Biol Cell, 15:5118-5129.

[37] Schulze-Lefert P. (2004) Curr Opin Plant Biol., 7:377-383.

[38] $\mathrm{Nu}$ " rnberger T. and Lipka V. (2005) Mol Plant Pathol., 6:335-345.

[39] Bonifacino J. S. and Glick B. S. (2004) Cell, 116:153-166.

[40] Hardham A. R., Takemoto D. and White R. G. (2008) BMC Plant Biol., 8:63.

[41] Weerasinghe R. R., McK Bird. D. and Allen N. S. (2005) Proc Natl Acad Sci U.S.A., 102:3147-3152.

[42] Rodriguez A. J., Shenoy S. M., Singer R. H. and Condeelis J. (2006) J Cell Biol., 175:67-76.

[43] Lee J, Rudd J. J., Macioszek V. K. and Scheel D. (2004) J Biol Chem., 279:22440-22448.

[44] Bhat R. A., Miklis M., Schmelzer E., Schulze-Lefert P. and Panstruga R.(2005) Proc Natl Acad Sci U.S.A., 102:3135-3140.

[45] Assaad F. F., Qiu J. L., Youngs H., Ehrhardt D., Zimmerli L., KaldeM., Wanner G., Peck S. C., Edwards H., Ramonell K., Somerville C. R. and Thordal-Christensen H. (2004) Mol Biol Cell, 15:5118-5129.

[46] Staiger C. J. (2000) Annu Rev Plant Physiol Plant Mol Biol., 51: 257-288.

[47] Wasteneys G. O. and Galway M. E. (2003) Annu Rev Plant Biol., 54: 691-722.

[48] Bonfante P., Bergero R., Uribe X., Romera C., Rigau J. and Puigdomenech $\mathrm{P}$. (1996) Plant J., 9: 737-743.

[49] Diaz E. C., Martin F. and Tagu D. (1996) Plant Mol Biol., 31: 905-910. 
[50] Niini S. S., Tarkka M. T. and Raudaskoski M. (1996) Physiol Plant, 96: 186-192.

[51] Timonen S. and Peterson R. L. (2002) Plant Soil, 244: 199-210.

[52] Timonen S., So" derstro"m B. and Raudaskoski M. (1996) Mycorrhiza, 6: 423-429.

[53] Genre A. and Bonfante P. (1998) New Phytol, 140: 745-752.

[54] Uetake Y. and Peterson R. L. (1997) Can J Bot., 75: 1661-1669.

[55] Matsubara Y., Uetake Y. and Peterson R. L. (1999) Can J Bot., 77: 1159-1167.

[56] Blancaflor E. B., Zhao L. and HarrisonM. J. (2001) Protoplasma, 217: 154-165.

[57] Armstrong L. and Peterson R. L. (2002) Mycologia, 94: 587-595.

[58] Cornelis G. R. and Gijsegem V. F. (2000) Annu Rev Microbiol., 54: 735-774.

[59] Bu" ttner D. and Bonas U. (2003) Curr Opin Plant Biol., 6: 312-319.

[60] Gala'n J. E. and Zhou D. (2000) Proc Natl Acad Sci USA., 97: 8754-8761.

[61] Shao F., Merritt P. M., Bao Z., Innes R. W. and Dixon J. E. (2002) Cell 109: 575588.

[62] Jackson R. W., Athanassopoulos E., Tsiamis G., Mansfield J. W., Sesma A., Arnold D. L., Gibbon M. J., Murillo J., Taylor J. D. and Vivian A. (1999) Proc Natl Acad Sci USA., 96: 10875-10880.

[63] Abramovitch R. B., Kim Y-J., Chen SR., Dickman MB. and Martin G. B. (2003) EMBO J., 22: 60-69.

[64] Hauck P., Thilmony R. and He S.Y. (2003) Proc Natl Acad Sci USA., 100: 85778582.

[65] Esseling J. J., Lhuissier F. G. P. and Emons A. M. C. (2004) Plant Cell, 16: 933-944.

[66] Riely B. K., Ane' J-M., Penmetsa RV. and Cook D. R. (2004) Curr Opin Plant Biol., 7: 408-413.

[67] Felle H. H., Kondorosi E., Kondorosi A. and Schultze M. (1998) Plant J., 13: 455463.

[68] Ca'rdenas L., Holdaway-Clarke T. L., Sa'nchez F., Quinto C., Feijo J.A., Kunkel J.G. and Hepler P.K. (2000) Plant Physiol., 123: 443-451.

[69] Shaw S. L. and Long S. R. (2003) Plant Physiol., 131: 976-984.

[70] Krause A., Lan Vo T. T. and Broughton W. J. (1997) Molecular Plant-Microbe Interactions, 10(3): 388-393.

[71] Norbert C. A., de Ruijter., Bisseling T. and Mie A. C. (1999) Molecular PlantMicrobe Interactions, 12(9): 829-832.

[72] Schmidta S. M. and Panstruga R. (2007) Physiological and Molecular Plant Pathology, 71(4-6):135-148.

[73] Altenbach D. and Robatzek S. (2007) MPMI, 20(9):1031-1039.
[74] J., Motteram, Küfner I., Deller S., Brunner F., Hammond-Kosack K. E., Nürnberger T. and Rudd J. J. (2009) Cell res., 22(7):790-799.

[75] Volker L. Ralph P. (2005) Current opinion in plant biology, 8:625-631.

[76] Chisholm S. T., Coaker G., Day B. and Staskawicz B. J. (2006) Cell, 124:803814. 